\title{
Desarrollo embrionario y larval del bolo Diplectrum radiale Quoy y Gaimard, 1824 (Pisces: Serranidae)
}

\author{
Embryonic and larval development of the Caribbean sand perch Diplectrum radiale \\ Quoy and Gaimard, 1824 (Pisces: Serranidae)
}

\author{
Pedro López ${ }^{1}$, Jesús Rosas ${ }^{2}$, Aidé Velásquez ${ }^{1}$, Tomas Cabrera ${ }^{1}$ y Carlos Maneiro $^{1}$ \\ ${ }^{I}$ Escuela de Ciencias Aplicadas del Mar. ${ }^{2}$ Instituto de Investigaciones Cientificas. \\ Universidad de Oriente, Núcleo de Nueva Esparta, Apartado Postal 788, Porlamar, Venezuela. \\ rosas@ne.udo.edu.ve
}

\begin{abstract}
Self-fecundation of Diplectrum radiale a synchronic hermaphrodite species, was induced through a manual process; the fertilization percentage was $86.92 \pm 2.75 \%$, producing spherical, floating eggs, with a centric oil drop, a reduced perivitelline space and a smooth envelop. Fertilized eggs had an average diameter of $0.67 \pm 0.02 \mathrm{~mm}$ and a oil drop with an average diameter of $0.13 \pm 0.01 \mathrm{~mm}$ at $26.41 \pm 0.25^{\circ} \mathrm{C}, \mathrm{pH} 8.84$ \pm 0.17 and $38 \pm 1 \mathrm{ppm}$ of salinity. The embryonic development was described, observing the first cleavage at $0 \mathrm{~h}: 36 \mathrm{~min}$ after fecundation at $27.25 \pm 0.50^{\circ} \mathrm{C}$ average temperature.

The embryonic development described was similar to other species with pelagic eggs. Hatching started $17 \mathrm{~h}$ : $23 \mathrm{~min}$ after fertilization at $27^{\circ} \mathrm{C}$. At $17 \mathrm{~h}: 50 \mathrm{~min}$ the larval total length was $1.33 \pm 0.02 \mathrm{~mm}$, the vitelline sac total length was $0.79 \pm 0.03 \mathrm{~mm}$ and the oil drop diameter was $0.14 \pm 0.01 \mathrm{~mm}$. Growth was constant, decreasing at $43 \mathrm{~h}$ when the vitelline sac was almost reabsorbed, restarting growing at $53 \mathrm{~h}$ when mouth opened.

The last embryonic phases were critical, with a percentage of non viable eggs of $18.89 \pm 1.92 \%$ at $25.80 \pm 0.27^{\circ} \mathrm{C}$, pH $6.78 \pm 0.08$ and $38 \pm 1 \mathrm{ppm}$ salinity. At temperatures higher than $28^{\circ} \mathrm{C}$ embryonic development anomalies were evident with a high percentage of non viable eggs. For this reason it was not possible to compare development between natural and controlled ambient.

Embryonic and larval development of $D$. radiale using the manual induction method permitted to obtain larvae from just one mature individual meaning a reduction in cost and time in producing fish larvae.
\end{abstract}

Key words: self-fecundation, embryonic and larval development, Diplectrum radiale, hatching induction, larva
Resumen.- Mediante proceso manual se realizó la autofecundación de Diplectrum radiale una especie hermafrodita sincrónica; el porcentaje de fertilización de $D$. radiale fue de $86,92 \pm 2,75 \%$, produciéndose huevos esféricos, flotantes, con una gota lipídica céntrica, un reducido espacio perivitelino y una envoltura lisa. El diámetro promedio del huevo fertilizado fue $0,67 \pm 0,02 \mathrm{~mm}$ y diámetro de la gota de aceite de $0,13 \pm 0,01$ $\mathrm{mm}$ a $26,41 \pm 0,25^{\circ} \mathrm{C}, \mathrm{pH} 8,84 \pm 0,17$ y salinidad $38 \pm 1 \mathrm{ppm}$. Se describió el desarrollo embrionario y larval, ocurriendo la primera división a las 0 h y 36 min después de la fecundación a una temperatura promedio de $27,25 \pm 0,50^{\circ} \mathrm{C}$.

El desarrollo embrionario descrito fue similar al de otras especies con huevos pelágicos. La eclosión se inició a las 17 h: 23 min después de la fertilización a $27^{\circ} \mathrm{C}$; a las $17 \mathrm{~h}: 50 \mathrm{~min}$ la longitud total de la larva fue $1,33 \pm 0,02 \mathrm{~mm}$, la longitud total del vitelo fue de $0,79 \pm 0,03 \mathrm{~mm}$ y el diámetro de la gota de aceite fue de $0,14 \pm$ $0,01 \mathrm{~mm}$. El crecimiento de la larva fue constante, disminuyendo de tamaño a partir de las $43 \mathrm{~h}$ cuando el saco vitelino estaba casi reabsorbido y reiniciando su crecimiento a las $53 \mathrm{~h}$ cuando ocurrió la apertura de la boca.

Las últimas fases fueron críticas, con un porcentaje de huevos no viables de $18,89 \pm 1,92 \%$ a $25,80 \pm 0,27^{\circ} \mathrm{C}, \mathrm{pH}$ de $6,78 \pm 0,08$ y una salinidad de $38 \pm 1 \mathrm{ppm}$. A temperaturas mayores de $28^{\circ} \mathrm{C}$ ocurrieron anomalías en el desarrollo embrionario con un alto porcentaje de huevos no viables, lo que impidió comparar el desarrollo embrionario entre ambiente controlado y natural.

El desarrollo embrionario y larval en $D$. radiale, utilizando el método de inducción manual, permitió obtener larvas a partir de un sólo reproductor, lo que significa una alternativa en la reducción de costos y tiempo en la producción de larvas de peces.

Palabras clave: Autofecundación, desarrollo embrionario y larval, Diplectrum radiale, inducción del desove, larva

\section{Introducción}

El género Diplectrum es hermafrodita sincrónico, endémico de América; en el Atlántico está representado por $D$. formosum, D. bivittatum y D. radiale, esta última se encuentra distribuida en las costas de Suramérica desde el Golfo de Venezuela hasta Sao Paulo, Brasil. Al parecer no se encuentra en las Antillas, y en Venezuela es una especie común a lo largo de toda la costa, en aguas neríticas con fondos blandos (Cervigón 1991). Sin embargo, no ha sido capturada en las áreas insulares oceánicas (Cervigón 1991). D. radiale presenta una talla común de $20 \mathrm{~cm}$ de longitud total y su talla máxima es de $26 \mathrm{~cm}$, su carne es de buena calidad y se consume en fresco (Cervigón et al. 1992). 
La reproducción del género y su relación con la primera madurez sexual, morfología gonadal, asimetría gonadal, época de desove, tipo de desove y fecundidad han sido referidas en D. formosum por Obando (1987), Obando \& León (1989). Manrique (1988) realiza la descripción embrionaria y prelarval de $D$. formosum, usando en la inducción al desove diferentes hormonas. Kendall (1984) da a conocer las descripciones de dos tipos de larvas ( 1 y 2) de Diplectrum recolectadas en el Océano Atlántico y en el Pacífico. La presente investigación tiene como objetivo realizar la autofecundación mediante proceso manual y la descripción embrionaria y larval de $D$. radiale.

\section{Materiales y Métodos}

Los ejemplares de Diplectrum radiale se capturaron entre las islas de Margarita y Cubagua $\left(10^{\circ} 48^{\prime \prime} 29^{\prime} \mathrm{N}\right.$ y $\left.64^{\circ} 13^{\prime \prime} 18^{\prime} \mathrm{W}\right)$, seleccionándose 10 peces reproductores, los que se midieron, pesaron y se colocaron en igual número de recipientes con $10 \mathrm{~L}$ de agua de mar filtrada y esterilizada mediante UV $\left(1,5 \mathrm{~L} \cdot \mathrm{min}^{-1}\right) \cdot \mathrm{La}$ autofecundación de $D$. radiale se realizó siguiendo el método de Woynarovich (1986). Individualmente a cada pez se le masajeó el abdomen, los productos sexuales (óvulos y espermatozoides) expulsados se recolectaron en un recipiente de 2,5 L. Transcurridos $20 \mathrm{~min}$ se procedió al lavado en agua de mar filtrada y selección de 20 huevos $\mathrm{L}^{-1}$, observándose constantemente al microscopio su desarrollo, desde el momento de la fertilización hasta la eclosión de las larvas. Se realizaron mediciones del diámetro del huevo y de la gota de aceite, tomándose fotografías de cada estadio del desarrollo embrionario, con un microscopio Olympus provisto de un ocular de 7X. La terminología utilizada para describir los diferentes estadios del desarrollo embrionario se basó en Damas et al. (1978) y Manrique (1988).

La descripción del desarrollo larval se realizó desde la eclosión hasta la reabsorción del saco vitelino y la posterior apertura de la boca. El comportamiento embrionario en el tiempo se realizó utilizando 30 recipientes de vidrio de 4,5 L, 15 a temperatura controlada $\left(24^{\circ} \mathrm{C}\right)$ y 15 a temperatura ambiente $\left(30^{\circ} \mathrm{C}\right)$; a estos recipientes se les colocó aireación moderada y luz continua. La temperatura fue medida con un termómetro de $0,1^{\circ} \mathrm{C}$ de precisión y el $\mathrm{pH}$ con un $\mathrm{pH}$ metro marca HI 8424.

La fase crítica del desarrollo embrionario fue estimada mediante un experimento en que se colocaron 20 huevos $\mathrm{L}^{-1}$ en fase de mórula en 24 recipientes de 4,5 $\mathrm{L}$ con un volumen de $3 \mathrm{~L}$ de agua de mar, los cuales fueron divididos en grupos de tres y observados a intervalos de $3 \mathrm{~h}$ cada uno según lo referido por Manrique (1988).

\section{Resultados y Discusión}

Los progenitores de Diplectrum radiale seleccionados $(21,14 \pm 2,34 \mathrm{~cm}$ y $141,29 \pm 68,84 \mathrm{~g})$ expulsaron los espermatozoides y luego los óvulos. Inmediatamente después de la fecundación, ocurrió la separación del corion y el ooplasma dando lugar al espacio perivitelino (Zanuy 1975; Manrique 1988). Hoar \& Randall (1969) demostraron que los progenitores de Serranus subligerius aislados individualmente produjeron espermatozoides y fertilizaban sus propios óvulos, estas mismas observaciones fueron demostradas en este experimento.

El porcentaje de fertilización fue de $86,92 \pm 2,75 \%$ con $13,08 \pm 0,25 \%$ de huevos no viables a $26,41 \pm$ $0,25^{\circ} \mathrm{C}$, pH de $8,84 \pm 0,17$ y salinidad de $38 \pm 1 \mathrm{ppm}$. Rosas et al. (1996) reportaron para $D$. formosum un porcentaje de fertilización del 93,75 \pm 4,37\% induciendo el desove con la hormona gonadotropina coriónica humana (HCG). En Epinephelus microdon el porcentaje de fertilización varió entre 32,6\% y $99,9 \%$ utilizando HCG (Tamaru et al. 1996). Tucker et al. (1996) refieren de 83 a $100 \%$ de fertilización en desoves voluntarios de E. striatus a temperaturas de 23,1 a $27,9^{\circ} \mathrm{C}$. En general, la fertilización en peces está estrechamente relacionada con la temperatura, así como el método de inducción o el tipo de hormona, notándose que en los desoves inducidos, los rangos de fertilización son amplios entre 32,6 y 99,9\% (Rosas et. al. 1996; Tamaru et al. 1996), mientras que en desoves voluntarios estos valores son más estrechos, debido a que las hembras pueden retener sus óvulos por un período de tiempo suficiente para conseguir la maduración final (Tucker et al. 1996). En el presente estudio, el porcentaje de fertilización estuvo relacionado con el método de inducción utilizado.

\section{Características del huevo fertilizado}

Los huevos de Diplectrum radiale se caracterizaron por ser esféricos, flotantes, transparentes, no adhesivos, telolecitos, con una sola gota de lípido concéntrica, un reducido espacio perivitelino y una envoltura lisa; estas características son comunes en huevos de la familia Serranidae (Zanuy 1975; Kendall 1984; Manrique 1988; Rosas et al. 1996). Los huevos recién fertilizados (Fig. 1A) presentaron un diámetro de $0,67 \pm 0,02 \mathrm{~mm}$ y la gota de aceite uno de $0,14 \pm 0,01 \mathrm{~mm}$ (Tabla 1). Manrique (1988) reportó para Diplectrum formosum valores de diámetro de huevos de 0,72 $\pm 0,09 \mathrm{~mm}$ y de la gota de aceite entre 0,11 y $0,12 \mathrm{~mm}$ utilizando 

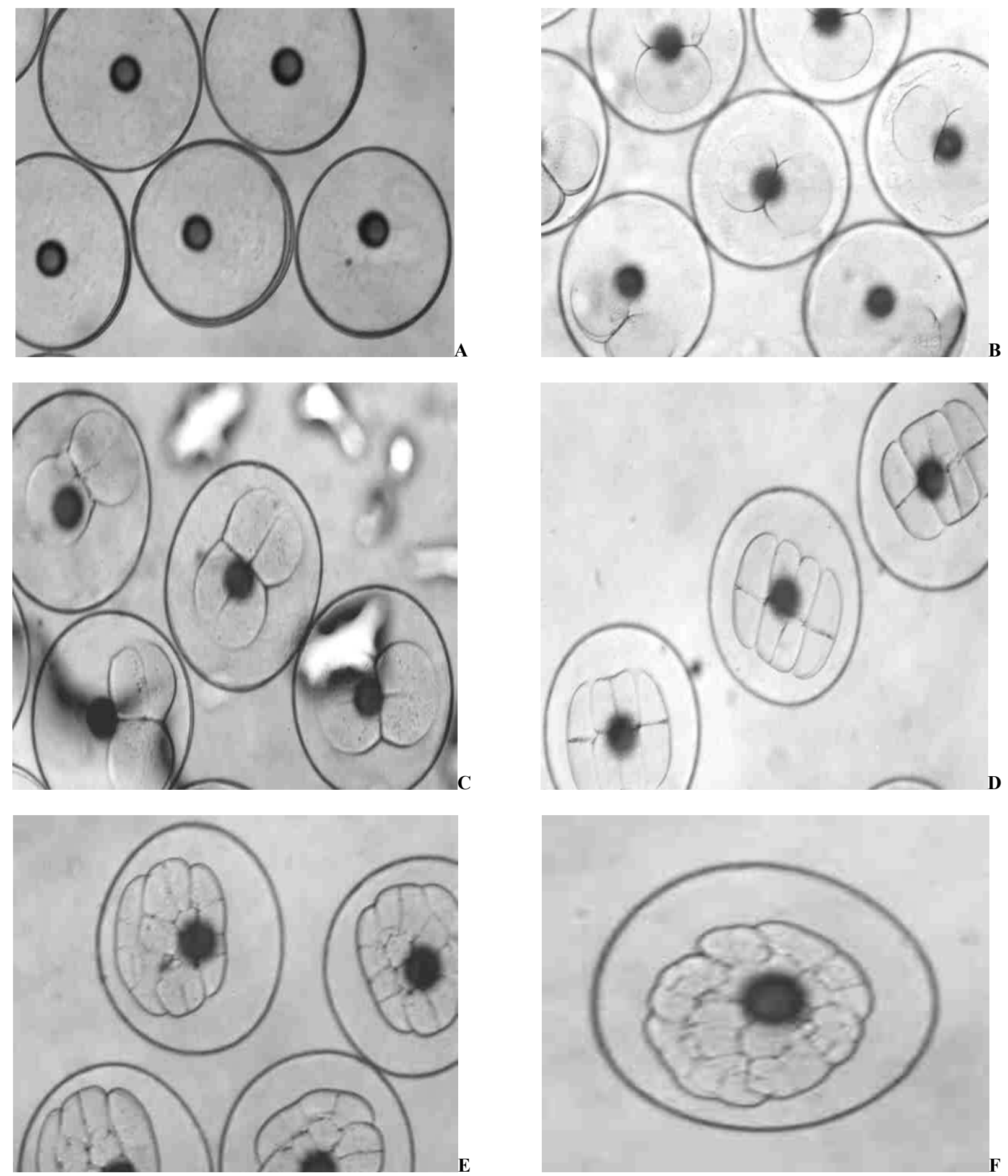

Figura 1

Desarrollo embrionario de Diplectrum radiale.

A) Huevo fertilizado; B) Primera división; C) Segunda división; D) Tercera división; E) Cuarta división; F) Quinta división Embryonic development of Diplectrum radiale.

A) Fertilized egg; B) First cleavage; C) Second cleavage; D) Third cleavage; E) Fourth cleavage; F) Fifth cleavage 
Tabla 1

Mediciones realizadas a los huevos y larvas de D. radiale a las 0, 24, 36, 43 y 53 horas después de la eclosión ( \pm desviación estándar), considerando diferentes características morfométricas

Eggs and larval measurements of Diplectrum radiale at 0,24, 36, 43 and 53 hours after hatching $( \pm \mathrm{SD})$, considering different morphometric characteristics

\begin{tabular}{cccccc}
\hline $\begin{array}{c}\text { Características morfométricas } \\
(\mathrm{mm})\end{array}$ & $0 \mathrm{~h}$ & $24 \mathrm{~h}$ & $\mathrm{~T} \mathrm{i} \mathrm{e} \mathrm{m} \mathrm{p} \mathrm{o}$ & \\
\hline LT & $1,33 \pm 0,02$ & $1,76 \pm 0,21$ & $1,95 \pm 0,18$ & $2,05 \pm 0,23$ & $1,57 \pm 1,08$ \\
LS & & & $1,84 \pm 0,18$ & $2,00 \pm 0,11$ & $1,03 \pm 1,05$ \\
LTV & $0,79 \pm 0,03$ & $0,72 \pm 0,05$ & $0,15 \pm 0,05$ & & \\
DGA & $0,14 \pm 0,01$ & $0,11 \pm 0,02$ & $0,11 \pm 0,03$ & & \\
DH & $0,70 \pm 0,02$ & & & &
\end{tabular}

LT: Longitud total; LS: Longitud estándar; LTV: Longitud saco vitelino; DGA: Diámetro gota de aceite; DH: Diámetro huevo

intervalos de temperatura de 23,5 a $24^{\circ} \mathrm{C}$ y 25 a $27,5^{\circ} \mathrm{C}$. Zanuy (1975) indicó que para Paracentropristis cabrilla los huevos presentaron un rango de diámetro comprendido entre $0,70-0,90 \mathrm{~mm}$ y la gota de aceite un rango de 0,14-0,15 mm. Butler et al. (1982) reportaron para los huevos de Paralabrax clathratus y $P$. maculatofasciatus un rango de 0,94 a $0,97 \mathrm{~mm}$ y de 0,80 a $0,89 \mathrm{~mm}$ y un diámetro de la gota de aceite de 0,20 y $0,17 \mathrm{~mm}$ respectivamente; lo que indica que el diámetro de los huevos fértiles varía con la especie. En tal sentido, Querales, (2001) refiere que los huevos fértiles de $P$. dewegeri presentan un diámetro de $0,89 \pm$ $0,02 \mathrm{~mm}$ y la gota de aceite $0,19 \pm 0,01 \mathrm{~mm}$, coincidiendo con Rosas et al. (1996).

\section{Etapa de división y formación del embrión}

El tiempo de ocurrencia de cada división a 27,25 \pm $0,50^{\circ} \mathrm{C}$ se presenta en la Tabla 2, Fig. 1B-F y Fig. 2A, concordando con los resultados obtenidos por Manrique (1988) con huevos de D. formosum a temperaturas entre 25,5 y $27,3^{\circ} \mathrm{C}$. El estadio de blástula (Fig. 2B) ocurrió a las $2 \mathrm{~h}$ : $33 \mathrm{~min}$ y a las $3 \mathrm{~h}: 24 \mathrm{~min}$ comenzó el de gástrula (Tabla 2, Fig. 2C-E). Manrique (1988) encontró para $D$. formosum que el estadio de blástula tuvo lugar a las $2 \mathrm{~h}$ : $40 \mathrm{~min}$, pero para la gástrula, el tiempo de ocurrencia fue de $5 \mathrm{~h}$ : $40 \mathrm{~min}$; esta diferencia en la aceleración del desarrollo embrionario en $D$. radiale, se relaciona con un incremento en la temperatura, lo que Zanuy (1975) atribuyó a un aumento en el metabolismo.

El engrosamiento del blastodisco (Fig. 2D), labio dorsal del anillo germinal, se observó a las 4 h: $02 \mathrm{~min}$. A las 6 h: 53 min se observó la presencia clara del esbozo embrionario. El tapón vitelino puede observarse inmediatamente antes que el vitelo quede encerrado por
Tabla 2

Resumen del desarrollo embrionario y larval del bolo Diplectrum radiale a $27,25 \pm 0,50^{\circ} \mathrm{C}$

Summary of embryonic and larval development of Caribbean sand perch Diplectrum radiale at $27,25 \pm 0,50^{\circ} \mathrm{C}$

\begin{tabular}{|c|c|}
\hline $\mathrm{h}: \min$ & Etapas del desarrollo desde la fecundación \\
\hline $0: 00$ & Fecundación \\
\hline $0: 36$ & Primera división \\
\hline $0: 42$ & Segunda división \\
\hline $0: 54$ & Tercera división \\
\hline 1: 07 & Cuarta división \\
\hline $1: 18$ & Quinta división \\
\hline $1: 36$ & Sexta división \\
\hline 2: 01 & Séptima división \\
\hline $2: 33$ & Blástula \\
\hline $3: 24$ & Gástrula \\
\hline $6: 53$ & Esbozo del embrión \\
\hline $8: 33$ & Inicio de la neurulación \\
\hline 10: 52 & $\begin{array}{l}\text { Esbozos ópticos y región caudal, embrión con } \\
7 \text { somitos y vesícula Kupffer }\end{array}$ \\
\hline $15: 54$ & $\begin{array}{l}\text { Movimiento del embrión, corazón produce de } \\
87 \text { a } 100 \text { latidos por minuto }\end{array}$ \\
\hline $17: 15$ & $\begin{array}{l}\text { Aumento del espacio entre el vitelo y el } \\
\text { corión, notocordio más visible }\end{array}$ \\
\hline $17: 23$ & Inicio de la eclosión \\
\hline $\mathrm{h}: \min$ & Etapas del desarrollo después de la eclosión \\
\hline 24: 00 & $\begin{array}{l}\text { Se observan cromatóforos a lo largo del } \\
\text { notocordio, esbozo del intestino }\end{array}$ \\
\hline 36: 00 & $\begin{array}{l}\text { Cápsulas ópticas, tubo gástrico y esbozo de la } \\
\text { boca mejor desarrollados }\end{array}$ \\
\hline 43: 00 & $\begin{array}{l}\text { Ojos con presencia de lentes, un cromatóforo } \\
\text { en la parte posterior de los mismos y una } \\
\text { marcada pigmentación oscura en esta región }\end{array}$ \\
\hline 53: 00 & $\begin{array}{l}\text { Apertura de la boca, larvas con gran } \\
\text { movilidad }\end{array}$ \\
\hline 72: 00 & Mortalidad del $95,80 \pm 0,67 \%$ de las larvas \\
\hline 80: 00 & Muerte del resto de las larvas \\
\hline
\end{tabular}


completo (Balinsky 1978). El proceso de gastrulación finalizó cuando se hizo visible el blastoporo y la banda embrionaria, la cual constituye el esbozo primario del embrión (Fig. 2F). Balinsky (1978) indica que en peces la formación de los esbozos primarios de los órganos se inicia desde la sección anterior a la posterior y los bordes laterales del blastodisco intervienen de modo gradual en la formación del cuerpo, los cuales se extienden hacia la línea media y contribuyen a la formación de las partes más posteriores del cuerpo y la cola.

\section{Etapa de desarrollo del embrión}

El estadio de néurula (Fig. 2F) se inició a las 8 h: 33 min; en esta etapa, persiste el tapón vitelino, permaneciendo el blastoporo aún sin cerrarse (Zanuy 1975). Según Rosas et al. (1996) en Paralabrax dewegeri el estadio de néurula ocurrió $8 \mathrm{~h}$ después de la fertilización, Manrique (1988) refiere que este estadio ocurre a las $8 \mathrm{~h}: 20 \mathrm{~min}$. En este trabajo, a las $10 \mathrm{~h}: 52$ min, se observaron claramente los esbozos ópticos y la región caudal; se distinguieron en el embrión 7 miotomos o somitas y la vesícula de Kupffer (Fig. 3A), que después del cierre del blastoporo se funde con los esbozos intestinales y hepáticos, lo que indicó una especie de sistema hepatopancreático propio del embrión en desarrollo (Zanuy 1975).

Rosas et al. (1996), referente a $P$. dewegeri, reportaron a las $9 \mathrm{~h}$ la vesícula de Kupffer y a las $9 \mathrm{~h}$ : 15 min los primeros somitas. En D. formosum, a las 10 h: 15 min se observaron los esbozos de las cápsulas ópticas, entre las cuales se va a ubicar el protocerebro y a las 11 h: 00 min se observó la vesícula de Kupffer (Manrique 1988). El final del estadio de néurula fue a las $15 \mathrm{~h}$ : $23 \mathrm{~min}$, notándose con claridad las cápsulas ópticas, la región caudal, leves movimientos en el embrión y un aumento en el número de somitas (Fig. 3B); movimientos amplios en el embrión se observaron a las 15 h: 54 min, así como 87 a 100 latidos por minuto en el corazón.

En Paralabrax dewegeri, las cápsulas y los primeros movimientos del embrión se observaron a las $10 \mathrm{~h}$ : 30 min después de la fecundación (Rosas et al. 1996); Manrique (1988) señala que en Diplectrum formosum, entre las 12 y $14 \mathrm{~h}$ se empiezan a distinguir claramente las cápsulas ópticas y se observó en el embrión un aumento en el número de somitas, a las $20 \mathrm{~h}$, se iniciaron los movimientos periódicos del embrión y el corazón latió entre 50 y 100 pulsaciones por minuto.

A las $17 \mathrm{~h}: 09$ min en la zona cefálica y caudal se notaron leves o pocos pigmentos de color rojo; en el saco vitelino los pigmentos presentaron una forma hexagonal y una coloración verde aguamarina traslúcida. A las 17 h: 15 min se observó un aumento del espacio entre el vitelo y el corion, así como la formación más visible del notocordio. Se observaron dos líneas formadas por cromatóforos de color rojo a ambos lados del notocordio y en la región cefálica. Las larvas de $D$. radiale iniciaron la eclosión a las 17 h: 23 min después de la fertilización (Tabla 2, Fig. 3C-E), a una temperatura de $27,0 \pm 1{ }^{\circ} \mathrm{C}$. En $D$. formosum la eclosión se inició a las $21 \mathrm{~h}$, a una temperatura entre 25,1 y $27,3^{\circ} \mathrm{C}$ (Manrique, 1988). Rosas et al. (1996) indicó que en Paralabrax dewegeri, la eclosión de la larva ocurrió a las $13 \mathrm{~h}$ a $29^{\circ} \mathrm{C}$; mientras que Querales (2001) señaló que las larvas de la misma especie eclosionaron a las $16 \mathrm{~h}: 20 \mathrm{~min}$ a los $27,0 \pm 1^{\circ} \mathrm{C}$.

Tamaru et al. (1996) señalan que larvas de Epinephelus microdon eclosionaron entre 18 y $21 \mathrm{~h}$ después del desove, a una temperatura de incubación entre 27 y $30^{\circ} \mathrm{C}$, e indican que el desarrollo embrionario fue rápido.

\section{Desarrollo larval}

La longitud total de las larvas recién eclosionadas (Fig. 3 F) fue de 1,33 $\pm 0,02 \mathrm{~mm}$, la gota de aceite se ubicó en la parte anterior y al transcurrir 15 min presentó un diámetro de $0,14 \pm 0,01 \mathrm{~mm}$, migrando hacia la parte posterior del saco vitelino, el cual presentó una longitud de 0,79 $\pm 0,03 \mathrm{~mm}$ (Tabla 1). Manrique (1988), señaló que las larvas de Diplectrum formosum midieron 1,36 \pm $0,66 \mathrm{~mm}$, el saco vitelino $0,76 \pm 0,50 \mathrm{~mm}$ y la gota de aceite se ubicó en la parte posterior del saco vitelino y midió $0,11 \pm 0,01 \mathrm{~mm}$, siendo estas larvas más grandes pero con el saco vitelino y la gota de aceite más pequeñas que las de $D$. radiale.

A las $24 \mathrm{~h}$ de eclosionadas (Fig. 4A), las larvas presentaron una longitud total de $1,76 \pm 0,21 \mathrm{~mm}$, la longitud del saco vitelino fue de $0,72 \pm 0,05 \mathrm{~mm}$ y el diámetro de la gota de aceite fue de 0,11 $\pm 0,02 \mathrm{~mm}$ (Tabla 1). Se observaron cromatóforos a lo largo del notocordio, siendo más abundantes en la aleta caudal, de color amarillo, además de resaltar tres cromatóforos negro rojizo, con una mancha negra en el centro. En la región dorsal y en la gota de aceite se identificaron cromatóforos marrones. El corazón se pudo observar, cuantificándose hasta 85 pulsaciones en 22 segundos. Se identificó claramente el esbozo del intestino, una marcada formación del orificio anal y la presencia de lentes en las cápsulas ópticas, siendo las larvas resistentes a la manipulación. 

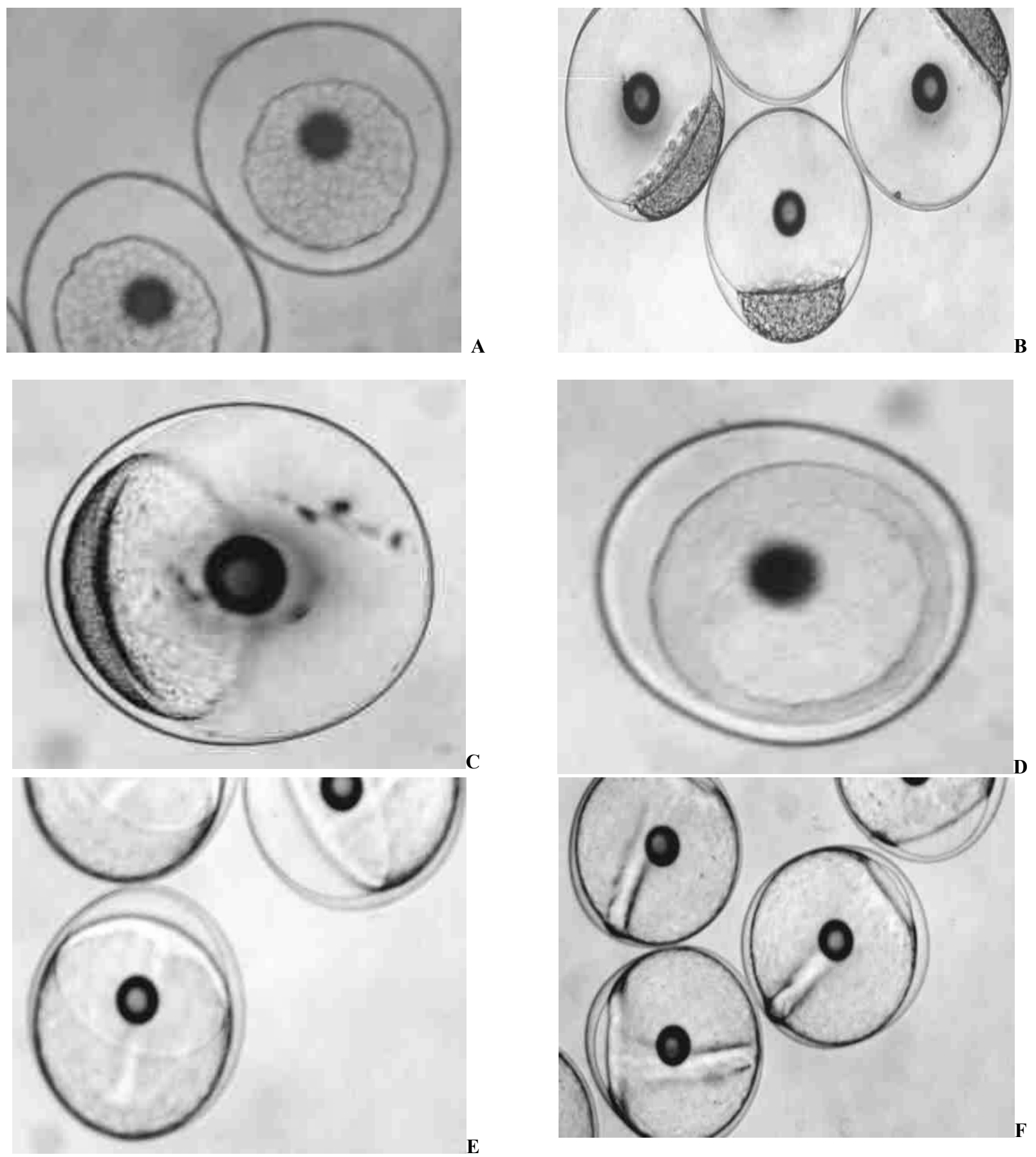

Figura 2

Desarrollo embrionario de Diplectrum radiale.

A) Mórula; B) Blástula; C) Gástrula temprana; D) Engrosamiento del blastodisco; E) Gástrula avanzada; F) Neúrula temprana

Embryonic development of Diplectrum radiale.

A) Morula stage; B) Blastula stage; C) Early gastrula; D) Blastodisc thickness; E) Advanced gastrula; F) Early neurula 

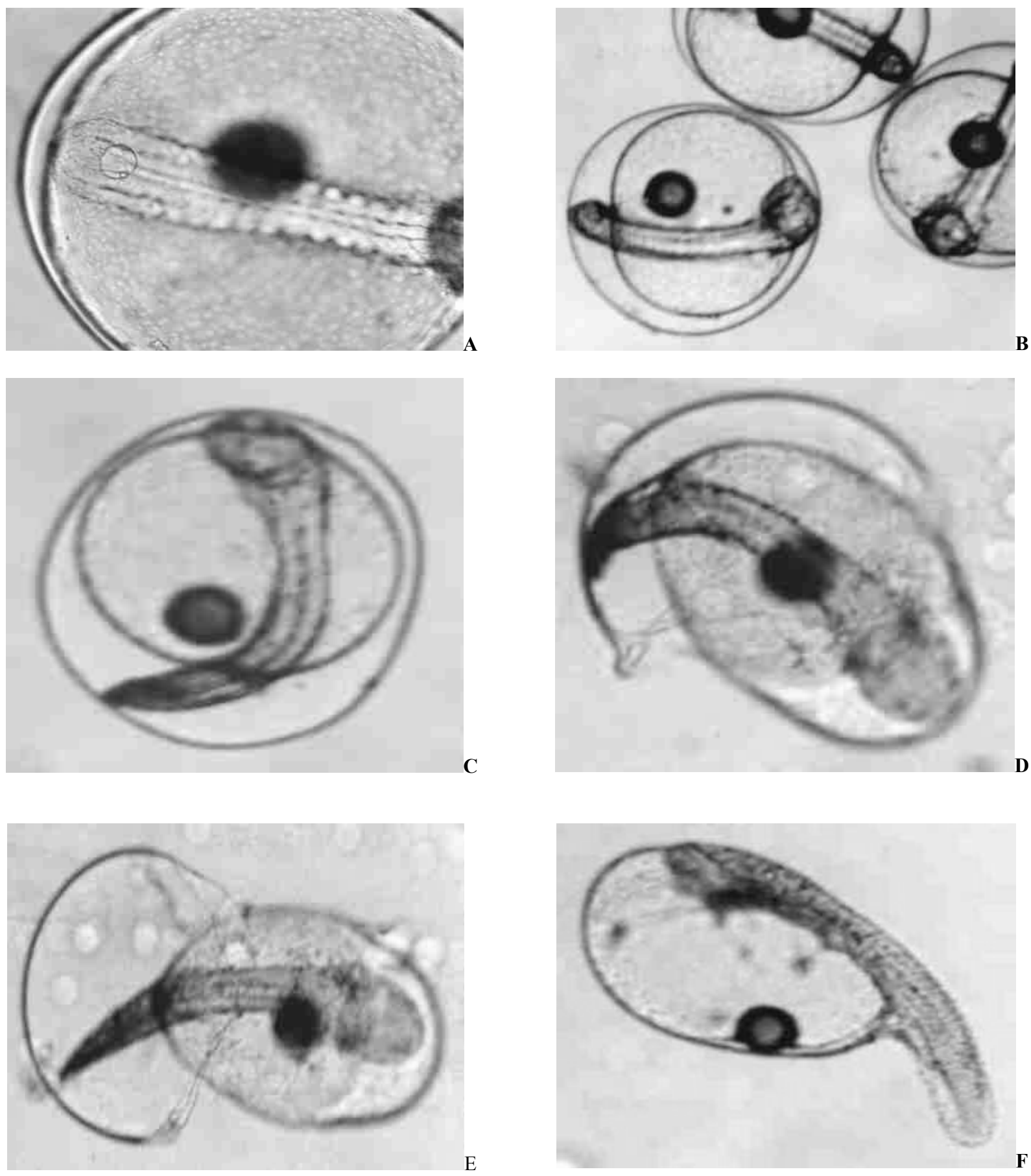

Figura 3

Desarrollo embrionario y larval de Diplectrum radiale.

A) Neúrula con vesícula de Kupffer; B, C) Larva próxima a eclosionar; D, E ) Larva eclosionando;

F) Larva recién eclosionada

Embryonic and larval development of Diplectrum radiale.

A) Neurula with Kupffer vesicle; B, C) Larva near to hatch; D, E ) Hatching larva;

F) Recently hatched larva 

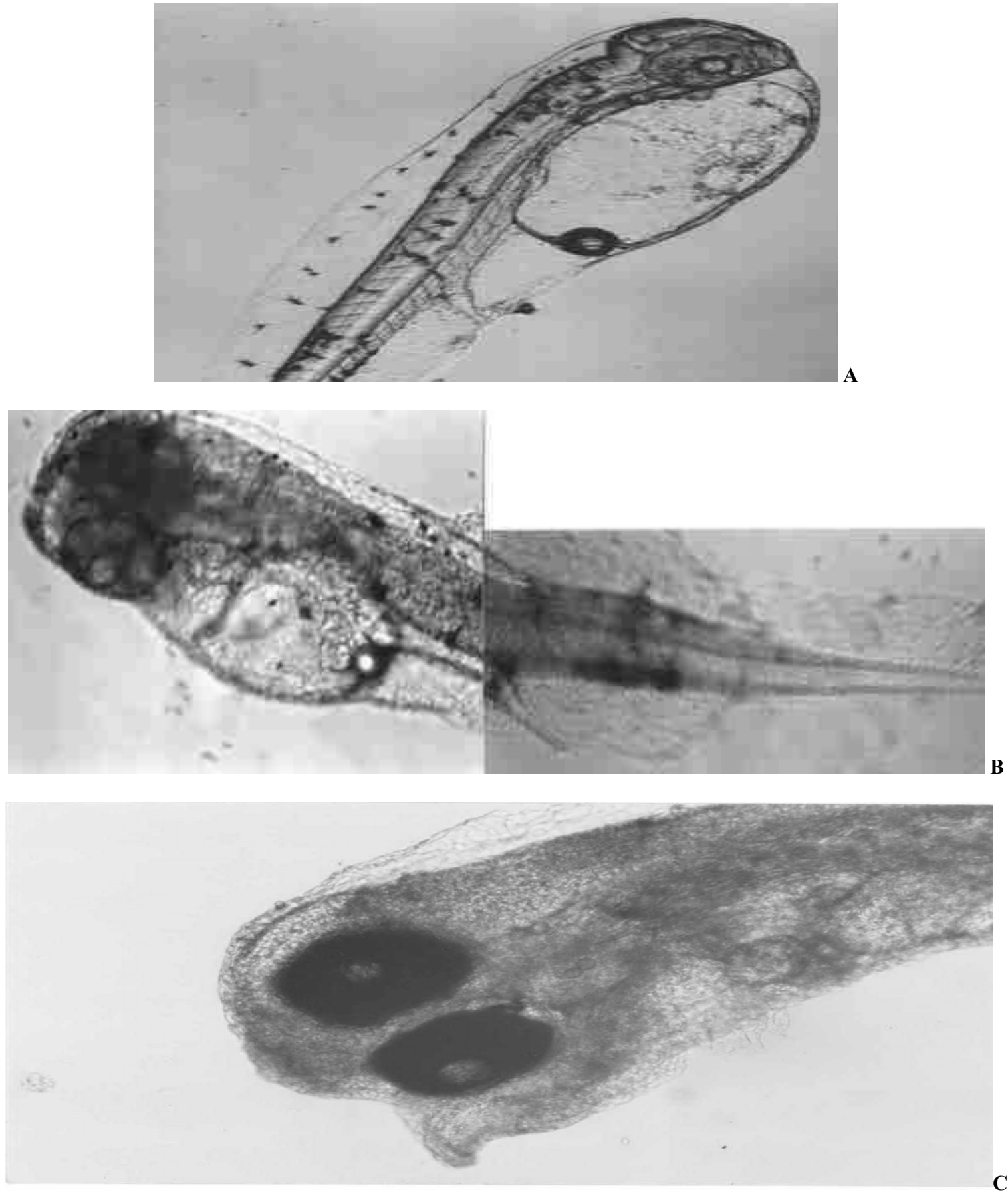

Figura 4

Larvas de Diplectrum radiale después de la eclosión.

A) de 24 horas; B) de $\mathbf{4 3}$ horas; C) de 53 horas

Diplectrum radiale larvae after hatching.

A) 24 hours old; B) 43 hours old; C) 53 hours old 
Manrique (1988) refirió que las larvas de $D$. formosum a las $24 \mathrm{~h}$ de eclosionadas midieron 1,75 \pm $0,08 \mathrm{~mm}$ y el saco vitelino $0,37 \pm 0,06 \mathrm{~mm}$; éstas presentaron igual tamaño que las larvas de Diplectrum radiale, con la diferencia de que el saco vitelino es de mayor tamaño en $D$. formosum.

A las 36 h de eclosionadas, el corazón incrementa el número de latidos y dificulta su conteo, las cápsulas ópticas, el tubo gástrico y el esbozo de la boca están mejor desarrollados; a nivel frontal se observaron dos cromatóforos; para este momento las larvas presentaron un tamaño promedio de $1,95 \pm 0,18 \mathrm{~mm}$, la longitud del saco vitelino fue de $0,15 \pm 0,05 \mathrm{~mm}$ y el diámetro de la gota de aceite fue de $0,11 \pm 0,03 \mathrm{~mm}$ (Tabla 1).

A las $43 \mathrm{~h}$ de eclosionadas (Fig. 4B) la longitud total fue 2,05 $\pm 0,23 \mathrm{~mm}$ y la longitud estándar de 2,00 \pm $0,11 \mathrm{~mm}$ (Tabla 1). La región cefálica más desarrollada, muestra los ojos con la presencia de lentes, un cromatóforo en la parte posterior de los mismos y una marcada pigmentación oscura en esta región. El volumen del saco vitelino disminuyó y la gota de aceite fue poco visible, lo que dificultó su medición; en esta zona se observaron pigmentos verdes y rojizos. El tubo digestivo y el orificio anal se observaron mejor desarrollados con pigmentación rojiza. La pigmentación general de las larvas fue verde y marrón en mayor cantidad a lo largo del cuerpo; los cromatóforos y los miómeros se observaron más acentuados. Las larvas nadan cerca de la superficie en posición diagonal con la cola en dirección hacia la superficie. A las $43 \mathrm{~h}$ de eclosionadas se observó una mortalidad masiva.

\section{Apertura de la boca}

La apertura de la boca de las larvas ocurrió a las $53 \mathrm{~h}$ de eclosionadas (Fig. 4C), siendo el tamaño de la mandíbula inferior de $0,19 \pm 0,01 \mathrm{~mm}$ y el alto de la boca de 0,27 $\pm 0,01 \mathrm{~mm}$; en estos momentos las larvas presentaron mayor movilidad, las aletas pectorales bien formadas con una longitud de $0,13 \pm 0,02 \mathrm{~mm}$ y las larvas con una longitud total de $1,57 \pm 1,08 \mathrm{~mm}$ y longitud estándar de 1,03 $\pm 1,05 \mathrm{~mm}$ (Tabla 1).

Según Manrique (1988), las larvas de D. formosum entre las 48 y $72 \mathrm{~h}$ después de la eclosión han reabsorbido completamente el material de reserva, la boca es móvil y la mandíbula inferior está perfectamente formada, presentando una longitud promedio de 2,30 $\pm 0,11 \mathrm{~mm}$ siendo de mayor tamaño que la de $D$. radiale.

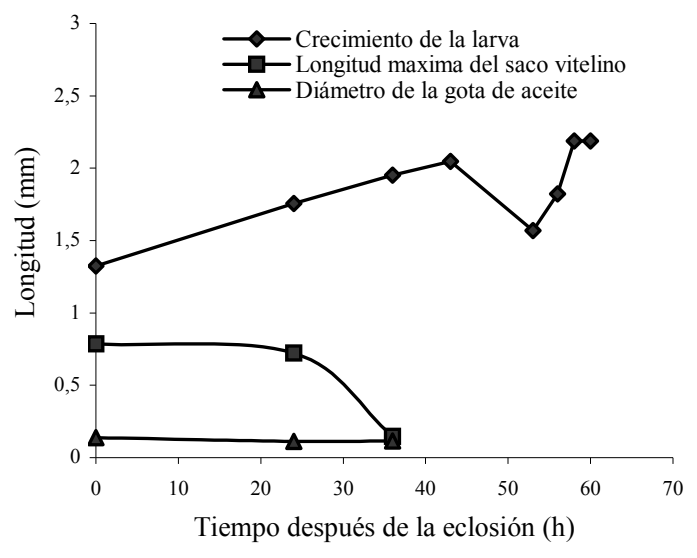

Figura 5

Comparación entre el crecimiento de las larvas, la longitud máxima del saco vitelino y el diámetro de la gota de aceite de Diplectrum radiale con respecto al tiempo

Larval growth comparing maximum length of yolk sac and oil drop diameter of Diplectrum radiale with respect to time

\section{Crecimiento de las larvas de $D$. radiale}

Desde el momento de la eclosión se observó un crecimiento constante relacionado directamente con el consumo del saco vitelino, luego fue disminuyendo a partir de las $24 \mathrm{~h}$ de eclosionadas, notándose que a las $43 \mathrm{~h}$, el saco vitelino fue reabsorbido casi en su totalidad. La larva reinició su crecimiento a las $53 \mathrm{~h}$ de eclosionadas cuando abrió la boca (Fig. 5). Al respecto, Borrrero et al. (1978) y Damas et al. (1978), señalan similar característica para las especies Lutjanus synagris y L. griseus, e infieren sobre un desvío energético utilizado para el crecimiento a la formación de sus estructuras cefálica y apertura bucal.

\section{Consumo del material de reserva en $D$. radiale}

El consumo de las reservas vitelinas en $D$. radiale, aumentó rápidamente durante las primeras horas de vida de las larvas, coincidiendo con un crecimiento acelerado, para ese mismo período. Estos resultados son característicos en larvas de teleósteos marinos (Borrero et al. 1978); en L. synagris, L. griseus y en Paralabrax dewegeri (Cabrera et al. 1997; Querales 2001).

Transcurridas $36 \mathrm{~h}$ de la apertura de la boca, se le suministró como alimento rotíferos (Brachionus plicatilis) y copépodos (Apocyclops distans), los cuales no fueron consumidos; a las $72 \mathrm{~h}$ después de la eclosión la mortalidad fue de $95,80 \pm 0,67 \%$; a las $80 \mathrm{~h}$ de eclosionadas el resto de las larvas murió por inanición. Manrique (1988) señala que uno de los problemas de la larvicultura de $D$. formusum es la apertura bucal 
extremadamente pequeña y registró mortalidades en el mismo lapso de tiempo que en esta investigación. Tucker et al. (1996) indicaron que la temperatura ambiente de $28,40 \pm 1,31^{\circ} \mathrm{C}$ y un $\mathrm{pH}$ de $6,84 \pm 0,09$ pueden causar mortalidades masivas.

\section{Conclusiones}

La fertilización de los huevos de Diplectrum radiale es posible a partir de un solo ejemplar sexualmente maduro, aprovechando su cualidad de hermafrodita sincrónico.

El porcentaje de fertilización de los huevos de $D$. radiale para esta experiencia fue de $86,92 \pm 2,75 \%$, con un porcentaje de huevos no viables de 13,08 $\pm 0,25 \%$.

Los huevos de $D$. radiale son esféricos, pelágicos, transparentes, no adhesivos, con una sola gota lipídica céntrica y una envoltura lisa. Su diámetro promedio fue de $0,67 \pm 0,02 \mathrm{~mm}$ y el diámetro de la gota de aceite de $0,14 \pm 0,01 \mathrm{~mm}$.

La eclosión se produjo a las 17 h: 23 min después de la fertilización a $27,25 \pm 0,50^{\circ} \mathrm{C}$, quedando el vitelo totalmente reabsorbido entre 43 y 53 h después de la eclosión, coincidiendo con la apertura de la boca.

El comportamiento del desarrollo embrionario de $D$. radiale en el tiempo, demostró que las últimas fases del desarrollo embrionario fueron críticas, presentando un porcentaje de huevos no viables de $18,89 \pm 1,92 \%$ a una temperatura promedio de $25,80 \pm 0,27^{\circ} \mathrm{C}$ y a un $\mathrm{pH}$ de $6,78 \pm 0,08$.

La longitud total de las larvas después de la eclosión fue de $1,33 \pm 0,02 ; 1,76 \pm 0,21 ; 1,95 \pm 0,18 ; 2,05 \pm$ 0,$23 ; 1,57 \pm 1,08 \mathrm{~mm}$ a las $0,24,36,43$ y $53 \mathrm{~h}$ respectivamente.

El porcentaje de mortalidad larval fue de 95,80 \pm $0,67 \%$ a las $72 \mathrm{~h}$ de eclosionadas y a las $80 \mathrm{~h}$ murió el resto.

\section{Literatura citada}

Balinsky B. 1978. Introducción a la embriología, 644 pp. Ediciones Omega S.A. Barcelona, España.

Borrero M, E González, N Millares \& T Damas. 1978. Desarrollo embrionario y prelarval de la biajaiba (Lutjanus synagris Linné, 1758). Revista Cubana de Investigaciones Pesqueras 3 (3): 1-12.
Butler JL, HG Moser, GS Hageman \& LE Nordgren. 1982. Developmental stages of three Californian sea basses (Paralabrax, Pisces: Serranidae). Californian Cooperation Oceanic Fish, Investment Report 23: 252 - 268.

Cabrera T, J Rosas \& J Millán. 1997. Reproducción y desarrollo larvario del pargo dienton (Lutjanus griseus L. 1758) (Pisces: Lutjanidae) cultivado en cautiverio. Caribbean Journal of Science 33 (3-4): 239-245.

Cervigón F. 1991. Los peces marinos de Venezuela. Fundación Científica de Los Roques. Caracas, Venezuela. 1: 315-321.

Cervigón F, R Ciprian, W Fischer, L Garibaldi, M Hendrickx, A Lemus, R Márquez, J Poutiers, G Robaina \& B Rodríguez. 1992. Guía de campo de las especies comerciales marinas y de aguas salobres de la costa septentrional de Sur América. Preparado con el Financiamiento de la Comisión de Comunidades Europeas y de NORAD, 531 pp. Roma, Italia.

Damas T, M Borrero, N Millares \& E González. 1978. Desarrollo embrionario y prelarval del caballote (Lutjanus griseus Linné, 1758). Revista Cubana de Investigaciones Pesqueras 3 (4): 11-21.

Hoar S \& D Randall. 1969. Fish Physiology. 3: 485 pp. Academic Press, Nueva York y Londres.

Kendall A. 1984. Serranidae: Development and relationships. En: Moser HD, Richards M, Fahay A \& S Richardson (Eds.) Ontogeny and systematics of fishes: 499-510. American Society of Ichthyologists and Herpetologists, Lawrence, Kansas.

Manrique R. 1988. Inducción a la puesta, desarrollo embrionario y prolarval de la yuqueta, Diplectrum formosum (Linnaeus: 1766). Trabajo de postgrado en Ciencias Marinas, 98 pp. Cumaná, Venezuela.

Obando E. 1987. Aspectos biológicos del bolo, Diplectrum formosum (Linnaeus, 1766), de la costa sureste de Margarita, Venezuela. Trabajo de postgrado en Ciencias Marinas, 169 pp. Cumaná.

Obando E \& J León. 1989. Reproducción del bolo, Diplectrum formosum (Linnaeus, 1766) (Pisces: Serranidae) en Punta Mosquito, Isla de Margarita, Venezuela. Scientia Marina 53 (4): 771-777.

Querales D. 2001. Descripción del desarrollo embrionario y larval de Paralabrax dewegeri Metzelaar, 1919 (Pisces: Serranidae). Trabajo para Licenciado, 71 pp. Boca de Río, Venezuela.

Rosas J, T Cabrera \& J Millán. 1996. Inducción al desove de peces marinos utilizando hormona gonadotropina coriónica humana. Proceedings of the $49^{\text {th }}$ Gulf and Caribbean Fisheries Institute: 46-51. 
Tamaru C, C Carlstrom-Trick, W Fitzgerald \& H Ako.

1996. Induced final maturation and spawnning of the marbled groupers Epinephelus microdon captured from spawning aggregations in the Republic of Palau, Micronesia. Journal of the World Aquaculture Society 27 (4): 363-372.

Tucker J, P Woodward \& D Sennett. 1996. Voluntary spawning of captive Nassau groupers Epinephelus striatus in a concrete raceway. Journal of the World Aquaculture Society 27 (4): 373-383.
Woynarovich E. 1986. Tambaqui e pirapitinga. Propagacao artificial e criacao de alevinos, 68 p. Programa de irrigacao-CODEVASF- Compahia de desenvolvimento do vale de Sao Francissco, Brazil.

Zanuy S. 1975. Desarrollo del huevo y estados larvarios de cabrilla (Paracentropristis cabrilla L.). Investigaciones Pesqueras 39 (2): 473-489. 